\section{Awareness of Scleroderma Peripheral Artery Disease Presenting With Critical Limb Ischemia in Elderly Patients}

Sayaka Funabashi, MD; Osami Kawarada, MD, PhD; Toru Hirano, MD, PhD; Shinobu Ayabe, MD; Takeshi Yagyu, MD; Teruo Noguchi, MD, PhD; Satoshi Yasuda, MD, PhD

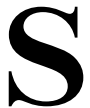
cleroderma is a slow progressive vascular disease with macrovascular manifestations in approximately in $15 \%$ of patients. ${ }^{1}$ An 80 -year-old woman with dyslipidemia was referred for treatment of a non-healing ulcer in the right first and fifth toes (Figure A). Right foot skin perfusion pressure was $17 / 16 \mathrm{mmHg}$ (dorsum/plantar), suggesting unlikely wound healing. Diagnostic angiography showed severe infrapopliteal artery disease with a type 1C variant (Figure B). Given the isolated infrapopliteal artery disease without diabetes mellitus and renal failure, non-atherosclerotic vasculopathy was highly suspected. Following a rheumatology consultation, the diagnosis of limited scleroderma was made with the evidence of anticentromere antibody 169 index (normal high $>16$ ), skin thickening and Raynaud's phenomenon. Medical treatment including antiplatelet agent and endothelin receptor antagonist failed to improve the clinical condition. Therefore, although surgical or endovascular recanalization might be controversial in the setting of vasculitis,, $\mathbf{2 3}$ endovascular recanalization was carried out. The occlusive anterior tibial and dorsalis pedis arteries were successfully recanalized to establish one straight-line flow, whereas the occlusive posterior tibial and plantar arteries were not (Figure C,D). The skin perfusion pressure increased to $32 / 20 \mathrm{mmHg}$ on the following day. The wound healed completely in 3 months with 3 clinically driven reinterventions and a scheduled minor amputation (Figure E). Histopathology of the amputated toe indicated concentric intimal hyperplasia leading to narrowing of the lumen in the digital artery, suggesting an underlying vasculitis (Figure F). Endothelin receptor antagonist as well as antiplatelet agent were given to prevent the recurrence of toe ulcer. During the last 6 months, no recurrence of the wound was observed.

This emphasizes the need for an increasing awareness of scleroderma-related peripheral artery disease (PAD) in elderly patients. Endovascular therapy might be a potential treatment option even in the setting of non-atherosclerotic PAD.

\section{Disclosures}

The authors declare no conflicts of interest.

\section{References}

1. Herrick A. Diagnosis and management of scleroderma peripheral vascular disease. Rheum Dis Clin North Am 2008; 34: 89-114.

2. Deguchi J, Shigematsu K, Ota S, Kimura H, Fukayama M, Miyata T. Surgical result of critical limb ischemia due to tibial arterial occlusion in patients with systemic scleroderma. $J$ Vasc Surg 2009; 49: $918-923$.

3. Obara H, Matsubara K, Fujimura N, Sekimoto Y, Kitagawa Y. Preliminary report of endovascular treatment for critical limb ischemia patients with connective tissue disease: Case series and review of literature. Int J Angiol 2015; 24: 137-142.

Received May 14, 2018; revised manuscript received June 24, 2018; accepted August 14, 2018; J-STAGE Advance Publication released online September 29, 2018 Time for primary review: 15 days

Department of Cardiovascular Medicine, National Cerebral and Cardiovascular Center, Suita (S.F., O.K., T.Y., T.N., S.Y.); Department of Respiratory Medicine, Allergy and Rheumatic Diseases, Osaka University Graduate School of Medicine, Suita (T.H.); and Department of Plastic Surgery, Yao Tokushukai General Hospital, Yao (S.A.), Japan

Mailing address: Osami Kawarada, MD, PhD, Department of Cardiovascular Medicine, National Cerebral and Cardiovascular Center, 5-7-1 Fujishiro-dai, Suita 565-8565, Japan. E-mail: kawarada. osami.hp@ncvc.go.jp

ISSN-1346-9843 All rights are reserved to the Japanese Circulation Society. For permissions, please e-mail: cj@j-circ.or.jp

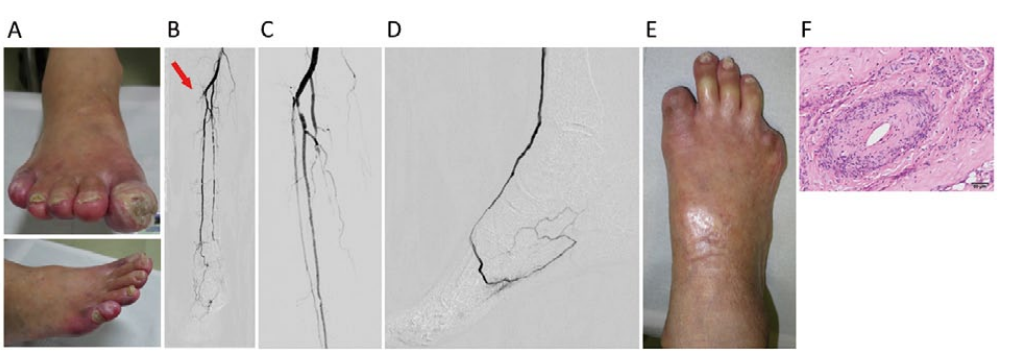

Figure. Scleroderma-related isolated infrapopliteal artery disease presenting with ischemic toe ulcer in an 80-year-old woman. (A) Non-healing ischemic ulcer on the right great and little toes. (B) Diagnostic angiography showed severe infrapopliteal artery disease with a type $1 \mathrm{C}$ variant consisting of an extensive occlusion from the ostium of the right anterior tibial artery to the dorsalis pedis artery (arrow), diffuse stenosis in the posterior tibial artery, and occlusion of the plantar artery. (C,D) Following balloon angioplasty of the anterior tibial and dorsalis pedis arteries, final angiography demonstrated an excellent straight-line flow to the foot and the establishment of pedal arch. The lesser visualization of the branches might be due to underlying small vessel disease or vasospasm related to vasculitis. (E) Complete wound healing after endovascular intervention and scheduled minor amputation. (F) Histopathology of the amputated toe showing concentric intimal hyperplasia leading to luminal stenosis or occlusion in the digital artery, suggesting an underlying vasculitis. 